\title{
BMJ Open Integrating clinical pharmacists within general practice: protocol for a pilot cluster randomised controlled trial
}

\author{
Aisling Croke, ${ }^{1}$ Frank Moriarty, ${ }^{1,2}$ Fiona Boland (D) , ${ }^{1,3}$ Laura McCullagh, ${ }^{4,5}$ \\ Karen Cardwell, ${ }^{1}$ Susan M Smith (D) , ${ }^{1}$ Barbara Clyne (i) ${ }^{1}$
}

To cite: Croke A, Moriarty F, Boland $\mathrm{F}$, et al. Integrating clinical pharmacists within general practice: protocol for a pilot cluster randomised controlled trial. BMJ Open 2021;11:e041541. doi:10.1136/ bmjopen-2020-041541

- Prepublication history and additional material for this paper are available online. To view these files, please visit the journal online (http://dx.doi. org/10.1136/bmjopen-2020041541).

Received 11 June 2020 Revised 11 February 2021 Accepted 16 February 2021

\section{Check for updates}

(C) Author(s) (or their employer(s)) 2021. Re-use permitted under CC BY-NC. No commercial re-use. See rights and permissions. Published by BMJ.

${ }^{1}$ Department of General Practice, Royal College of Surgeons in Ireland, Dublin, Ireland

${ }^{2}$ School of Pharmacy and Biomolecular Sciences, Royal College of Surgeons in Ireland, Dublin, Ireland

${ }^{3}$ Data Science Centre, Royal College of Surgeons in Ireland, Dublin, Ireland

${ }^{4}$ National Centre for

Pharmacoeconomics, St James's Hospital, Dublin, Ireland ${ }^{5}$ Department of Pharmacology and Therapeutics, Trinity College Dublin, Dublin, Ireland

Correspondence to

Aisling Croke;

aislingcroke@rcsi.com

\section{ABSTRACT}

Introduction Managing patients with multiple conditions (multimorbidity) is a major challenge for healthcare systems internationally, particularly in older patients. Multimorbidity and subsequent polypharmacy increase treatment burden and the risk of potentially inappropriate prescribing, and both are complex to manage in primary care. Limited evidence suggests integration of pharmacists into general practice teams could improve medication management for patients with multimorbidity and polypharmacy. Building on findings from a nonrandomised, uncontrolled General Practice Pharmacist (GPP) feasibility study conducted in Irish primary care, the aim of this study is to conduct a pilot cluster randomised controlled trial (cRCT) of the GPP study, to assess feasibility, intervention impact, costs and appropriateness of continuing to a definitive CRCT.

Methods and analysis This pilot CRCT will involve 8 general practitioner (GP) practices and 120 patients. Practices will identify and recruit patients aged $\geq 65$ years, who are taking $\geq 10$ regular medications. Practices will be allocated to intervention or control after baseline data collection. Intervention practices will have a pharmacist integrated within their service, working with GPs, patients and practice staff to optimise prescribing and other medication-related activities. Control practices will provide standard GP care. The primary feasibility outcomes will include recruitment rate, uptake of medication reviews and study retention. For the primary clinical outcome, the number of potentially inappropriate prescribing incidences per patient will be collected. Secondary outcomes will include medication-related outcomes, patient-reported outcome measures, and data pertaining to the role and impact of the pharmacist on prescribing. In addition, economic and process evaluations will be conducted. Ethics and dissemination This trial has been approved by the Irish College of General Practitioners Research Ethics Committee and will be performed in accordance with the Declaration of Helsinki. The results will be reported in peer-reviewed journals and be presented at national and international conferences.

Trial registration number ISRCTN Registry (https://doi. org/10.1186/ISRCTN18752158).

\section{INTRODUCTION}

Managing patients with multiple conditions (multimorbidity) and associated

\section{Strengths and limitations of this study}

This is one of the first pilot cluster randomised controlled trials (CRCTs) examining the feasibility and impact of the role of general practice-based pharmacists in Irish primary care.

- As all practices will be recruited from the Health Research Board Primary Care Clinical Trials Network Ireland, patients will be randomly selected to reduce the element of bias within the selection of general practitioner (GP) practices.

- Although the sample size is small (8 GP practices and 120 patients), sufficient data will be collected to determine feasibility and explore outcome selection for a possible future definitive trial.

- Generalisability is a limitation of this study as only eight practices will be included, but purposive sample of practices will be undertaken to ensure broad representation.

- Predefined continuation criteria based on recruitment, retention, intervention acceptability, feasibility and cost-effectiveness will inform potential trial progression to a definitive pragmatic CRCT.

polypharmacy is recognised as a major challenge for healthcare systems. A recent systematic review of 70 studies reported a $33 \%$ prevalence of multimorbidity (defined as two or more chronic conditions) in community settings, with a large proportion (more than $50 \%$ in many cases) of individuals aged 65 years and over having multimorbidity. ${ }^{1}$ The volume of medications being prescribed to older patients has increased over the past number of years also. A study analysing prescribing trends over a 15 -year period found that polypharmacy (commonly defined as patients taking five or more regular medications) affects $60.4 \%$ of the Irish population aged 65 years or older. ${ }^{2}$ Complex polypharmacy, defined as patients taking 10 or more regular medications, affects $21.9 \%$ of Irish patients. ${ }^{2}$ Polypharmacy is often associated with multimorbidity as best practice and clinical guidelines are typically derived 
from populations which do not reflect these patients and tend to be single disease focused. ${ }^{3}$ Overall management of these multiple medications, commenced by various specialists, is usually provided by a patient's general practitioner (GP). There is an increased medication burden in this patient cohort and this leads to the risk of potentially inappropriate prescribing (PIP). PIP can be described as suboptimal prescribing, and may lead to adverse drug reactions (ADRs), ${ }^{4}$ hospitalisations and subsequent costs. Older people are most at risk of negative consequences of polypharmacy ${ }^{5}$ and as such, prescribing screening tools typically focus on medications in older patients. ${ }^{6}$ An Irish study conducted in 2010 found that an estimated 36\% of adult patients over the age of 70 years had at least one PIP event. This resulted in an additional $€ 45$ million in healthcare expenditures. ${ }^{7}$

Within this context it is important to develop interventions for patients with multimorbidity and polypharmacy which reduce PIP as a public health measure. Interventions to improve appropriate prescribing include those aimed at prescribers (eg, computerised decision support), patient education and changes to care delivery arrangements, such as staffing models or skills-mix. ${ }^{89}$ One such intervention is the integration of clinical pharmacists into general practices to address PIP, deprescribing, medication reviews and general practice workload..$^{10}$ Evidence suggests that pharmacists in general practice can have positive impacts on clinical outcomes with one systematic review reporting a significant reduction in glycated haemoglobin between pharmacist intervention groups and control (mean difference $-0.88 \%, 95 \%$ CI $-1.15 \%$ to $-0.62 \%, \mathrm{p}<0.001)^{1011}$ and prescribing safely. In particular, the PINCER trial in the UK demonstrated that a pharmacist-led information technology intervention was an effective method for reducing a range of medication errors in general practice. ${ }^{12}$ Integrating pharmacists into primary care may also reduce GP workload (particularly medication-related administration), emergency department attendance and medication-related hospitalisations. ${ }^{13}$ The current evidence base is however varied with some high-quality studies such as the PINCER trial demonstrating effectiveness, ${ }^{12}$ and some smaller studies of mixed quality with mixed results. Further high-quality research is needed to assess the impact of this new role on patient outcomes, GP workload, stakeholder experiences and cost-effectiveness. ${ }^{11} 13$

\section{Rationale for study}

Unlike countries such as the UK and Canada, pharmacists in Ireland are not formally integrated into general practice, nor do they have prescribing rights. Primary care in Ireland is delivered in a mixed public and private healthcare system, described in more detail in online supplemental appendix 1. The General Practice Pharmacist (GPP) feasibility study demonstrated that an intervention involving pharmacists working within general practices in Ireland is feasible to implement and has potential to improve prescribing quality in older patient populations. ${ }^{14-16}$

The aim of this study is to conduct a pilot cluster randomised controlled trial (cRCT) of the GPP study, to assess feasibility, potential intervention impact and costs, and assess whether it is appropriate to continue to a definitive cRCT.

\section{Development of the GPP Medicines Optimisation Study}

This pilot cRCT has been informed by the nonrandomised, uncontrolled feasibility study ${ }^{16}$ as outlined in table 1. All but two of the continuation criteria from the uncontrolled feasibility study reached the threshold of 'Proceed with RCT'. ${ }^{16}$ Based on these findings and the process evaluation, areas highlighted for change to improve the pilot cRCT included (table 1):

1. Patient recruitment.

2. Evaluating the role and impact of the pharmacist when integrated within the GP practice.

3. Pharmacist isolation in GP practice.

4. Issues surrounding space in GP practices and individual case discussion time allocation with GPs.

5. Immediacy of communication with GPs.

6. Standard framework for medication review.

\section{METHODS/DESIGN}

\section{Objectives of study}

This study will involve the conduct and evaluation of a pilot cRCT of an intervention of clinical pharmacists based in general practice, to determine if general practice-based pharmacists are feasible and can potentially improve the management of, and outcomes for, patients with complex polypharmacy, in comparison with usual GP care.

The pilot cRCT will also inform the conduct (using formal continuation criteria) and sample size of a definitive trial.

\section{Study design}

A cluster design was chosen to address potential contamination of GPs as a result of exposure to working with a pharmacist during the intervention. GP practices are the units of randomisation (the clusters), and individual patients with polypharmacy are the units of analysis (the participants) with adjustments for clustering. We will report the trial according to the Consolidated Standards of Reporting Trials guidelines adapted for pilot studies. ${ }^{17}$

The pilot cRCT design was informed by the Medical Research Council (MRC) Framework for the design and evaluation of complex interventions. ${ }^{18}$ Informed consent will be obtained from participants (practices and patients) prior to data collection.

\section{Study setting}

This study will take place in the Irish General Practice setting. Both single-handed and group practices from the Health Research Board (HRB) Primary Care Clinical Trials Network Ireland (PC CTNI) (http://primarycaretrials.ie) will be included. 
Table 1 Influence of the feasibility study on the pilot $\mathrm{CRCT}$

Study domain

Patient recruitment

Examine pharmacist role in the GP practice
Lessons learnt from uncontrolled feasibility study in 4 practices and adaptations for proposed pilot CRCT

Target patient recruitment numbers for the feasibility study; $n=200,50$ patients in each of the practices $(\mathrm{n}=4)$.

Recruitment proved challenging for the feasibility study. It was anticipated that there would be no difficulty in recruiting 50 patients per practice given the known prevalence of polypharmacy, however recruitment was challenging within the short time frame for the feasibility study, which was only 10 weeks. Patients were recruited from month 4 to month 6 of the 6 -month study. This did not leave sufficient time to recruit the requisite numbers.

Patients did not understand the role of the pharmacist in the GP practice during the feasibility study. Patient engagement is very important in terms of the interventions acceptability to all stakeholders. This was a possible influencing factor in the lower than expected recruitment rates in the feasibility study and highlighted a need to prepare patients over time for new approaches to clinical care.

Adaptations for proposed pilot CRCT:

Target patient recruitment numbers for the pilot $\mathrm{cRCT} ; \mathrm{n}=120,15$ patients per practice.

This cohort of patients suffers from significant treatment burden and would likely attend a number of visits to various healthcare professionals. It may be that patients did not want to attend another appointment in relation to their care. The target number of patients to be recruited was reduced for the pilot $\mathrm{cRCT}$ based on the experience in the feasibility study. A sample size calculation was performed which was powered to detect a significant change in PIP.

It is possible that uptake would have been higher in the feasibility study had there been sufficient time to schedule the medication review with the pharmacist alongside a patient's 3-monthly or 6-monthly review with the GP.

Recruitment will thus begin before the pharmacist integrates into the practice. This will allow more time for recruitment and help to plan the workload of the pharmacist when in the practice. It may also allow for the scheduling of medication reviews alongside GP appointments to decrease the burden of attending on patients.

Patient education on the role of the pharmacist will be important in determining the value the patient can get from using the pharmacist as an appropriate resource. How this information is best disseminated was brought to a 'patient and public involvement' panel which had been set up to advise this PhD project alongside three other projects, which are funded through a specific multimorbidity PhD programme. Members of this panel are patients who live with multimorbidity and provide a wealth of experience and information in an advisory capacity.

Three main methods of patient education surrounding how they would like to learn of the pharmacist role emerged from that discussion.

1. From their GP.

2. From the pharmacist in the practice.

3. Poster on the waiting room wall in GP practice.

These methods will be employed to inform patients in the practice of the pharmacist role while based in the GP practice.

It is anticipated that these changes will enable patient recruitment during the intervention period.

The feasibility study highlighted a need to examine the role the pharmacist assumed in the practice in greater detail. The pharmacists in the feasibility study took part in a wide range of activities involving quality of practice, administration, medication review and education.

\section{Adaptations for proposed pilot CRCT:}

This proposed study will also evaluate the role and impact of a pharmacist on care provision within the general practice when integrated into the practice team in more detail than previously. As per the previous study, qualitative interviews will be conducted with participants. Additionally, a description of the activities that the pharmacist undertakes and the length of time undertaken to complete those activities will be collected. For the pilot CRCT, a pharmacist activity log will be used to capture pharmacist activities. This activity log will be updated weekly by the research pharmacists, and will not include any identifiable patient data. The purpose of the activity log is to document pharmacist tasks and actions, which will vary depending on practice requirements. This is key information in terms of implementation of this type of intervention.

The pharmacists will be joining the practice in this study as healthcare professionals, and are bound legally and ethically by the 'Code of Conduct' as presented by the Pharmaceutical Society of Ireland. ${ }^{38}$ They would be considered a member of the practice team for the duration of the intervention and will enter into a confidentiality agreement with the individual practices. As with any healthcare professional contributing to care, these factors would ensure confidentiality of individual patients' information. 
Table 1 Continued

Study domain

Pharmacist isolation in GP practice

Issues surrounding space in GP practices and time with GPs

Immediacy of communication with GPs

Lessons learnt from uncontrolled feasibility study in 4 practices and adaptations for proposed pilot CRCT

Pharmacist isolation was a theme identified during the feasibility study.

Adaptations for proposed pilot CRCT:

For the pilot CRCT, integrative workshops will be conducted with the practices to ensure the pharmacist is familiar with the practice, staff and operating systems. These workshops will provide an opportunity to educate practice staff as to the role of the pharmacist in the practice and identify the pharmacist as a resource for all members of the team, as in the feasibility study some staff stated they were not aware of this. The content of the workshops will be designed to introduce the pharmacist to the team and to meet the needs of the practice.

All activities related to these workshops will be recorded using the pharmacist activity log. The impact of education sessions such as this integrative workshop will be assessed in the post-intervention interviews.

This will be addressed by forward planning with the practices.

Adaptations for proposed pilot CRCT:

The research team will engage with practice staff to examine which practice staff will be present or absent during the intervention period and also if any movement of premises is planned. This will coordinate GP and pharmacist time to avoid any potential additional work burden to either parties.

Immediacy of query resolution was a factor for GPs during the feasibility study.

Adaptations for proposed pilot CRCT:

It is suggested for the pilot CRCT that practices can arrange with the pharmacist how best to contact them when they are not in the practice. In clinical practice, it is not always possible for clinicians to make immediate contact with each other but this issue will be raised as important during the pharmacist and practice training for the intervention.

$\mathrm{cRCT}$, cluster randomised controlled trial; GP, general practitioner; PIP, potentially inappropriate prescribing

\section{Trial status}

The trial is ongoing; practice and patient recruitment to commence in March 2021.

Anticipated intervention completion is in November 2022.

\section{Population and recruitment}

We will enrol a total of eight practices and each practice will recruit 15 patients (a total of 120 patients) with complex polypharmacy. All practices from the PC CTNI will be invited to participate via an email (or letter where email address is unavailable). Among eligible practices that express an interest in participating, we will recruit a purposive sample reflecting different practice sizes and locations (urban and rural).

\section{Clusters (GP practices)}

Practices will be eligible to participate if they have at least 500 older patients (aged $\geq 65$ years) on their patient panel to ensure adequate numbers of eligible patients and can use a GP software finder tool to identify patients with complex polypharmacy.

\section{Exclusion criteria}

Practices will be excluded if they have $<500$ older patients.

When a practice agrees to participate, practice staff will identify a list of potentially eligible patients using an electronic record finder tool that can identify patients on 10 or more regular medications. Practice staff, supported by the research team, will run this search to identify eligible patients. The practice will use a random number generator in Excel to identify a random selection of 15 eligible patients. Patients will continue to be randomly selected from the list of eligible patients and invited to take part until the full complement of 15 participants has been reached. Practices will send eligible patients a patient information pack containing a letter of invitation on practice headed paper, a patient information leaflet, a consent form and a patient questionnaire. A stamped addressed envelope for return of consent form and patient questionnaire to researchers will also be provided.

\section{Participants (patients)}

Patients will be eligible if they are 65 years of age or older, have complex polypharmacy (defined as $\geq 10$ repeat medications) and must have an ability to attend a medication review with the pharmacist. PIP is highly prevalent in older patient populations and thus this patient group is most at risk of negative consequences of polypharmacy. The implicit and explicit inappropriate prescribing screening tools used in this study have been designed for older patients and alternative indicators would need to be used in younger populations.

\section{Exclusion criteria}

- Under the age of 65 years.

- Terminally ill leading to high likelihood of death or major disability during study follow-up period, as judged by the patient's GP.

- Severe cognitive impairment, or psychiatric/psychological morbidity sufficient to impair informed consent, as judged by the patient's GP.

- Resident in nursing home. 


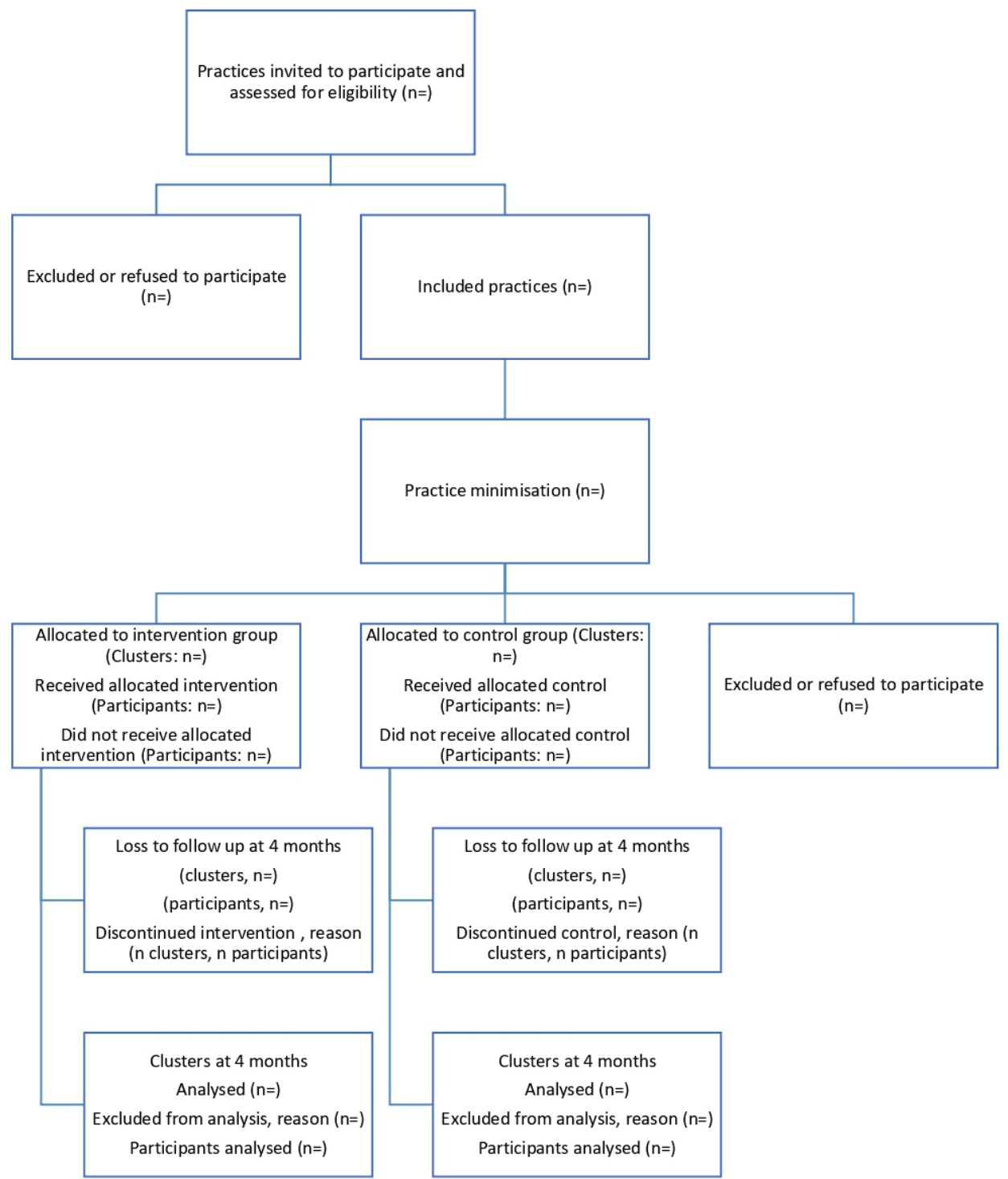

Figure 1 Flow of practices through the GPP Medicines Optimisation Programme pilot cRCT. cRCT, cluster randomised controlled trial; GPP, General Practice Pharmacist.

Currently participating in a related study.

To ensure transparency, a highly visible poster will be displayed in the practices and an information leaflet will be available outlining the role of the pharmacist, and also steps to take if an individual does not want their clinical information reviewed by the pharmacist.

\section{Randomisation}

Once consent and all baseline data collection has been completed, practices will be allocated into control or intervention groups using minimisation (see figure 1). This approach offers the advantage of ensuring balance between the groups in terms of prognostic factors, in this case, practice size (number of whole-time equivalent GPs) and practice location. Sequence generation and practice allocation will be carried out remotely by an independent statistician. Allocation of practices will be done in blocks to enable the pharmacists to deliver the intervention in different practices and stagger data collection.

\section{Intervention}

Pharmacists will be recruited via a competitive interview process. All pharmacists who currently operate in a clinical role will be considered eligible for interview, though it will be more desirable for the recruited pharmacists to have experience of working in community care and primary care settings. Two pharmacists will work between four intervention practices (two intervention practices per pharmacist). It is anticipated that the split of GP practices will be on an urban/rural basis and will be flexible to fit with pharmacist requirements. Intervention delivery will be staggered in practices. The pharmacist will integrate into the GP practice as per practice preference regarding time allocation for 10 hours per week for a period of 4 months. Integrative workshops prior to pharmacist commencement will ensure they are familiar with the practice, staff and operating systems. Written training material based on the previous feasibility study will be 
Table 2 Table 2 Proposed activities for pharmacists in GP practice

\begin{tabular}{|c|c|}
\hline Proposed activities & Potential activity \\
\hline - Clinical audit & $\begin{array}{l}\text { Topic requested by GP } \\
\text { Area determined by pharmacist based on analysis of aggregate data } \\
\text { As part of chronic disease management } \\
\text { Practice CPD requirements }\end{array}$ \\
\hline - Medication review & Review complex patients with GP and with patients themselves \\
\hline Repeat prescriptions management & $\begin{array}{l}\text { Medication review } \\
\text { - Discuss dose adjustments } \\
\text { - Medication initiation or discontinuation } \\
\text { - Medication that require extra monitoring }\end{array}$ \\
\hline - Education sessions & $\begin{array}{l}\text { Therapeutic area of interest } \\
\text { - GP directed } \\
\text { - Pharmacist directed } \\
\text { - Practice staff directed }\end{array}$ \\
\hline - Query management & $\begin{array}{l}\text { Phone call communication when not in practice to ensure immediacy of } \\
\text { query resolution } \\
\text { - GP queries } \\
\text { - Patient queries } \\
\text { - Other healthcare professional queries }\end{array}$ \\
\hline
\end{tabular}

provided to pharmacists also. When in the practices, pharmacists will support prescribing-related activities for consented patients as agreed with the GPs. They will also be available to support other related activities such as practice education and support with practice audits as requested. Table 2 describes in more detail the potential activities the pharmacist may undertake in the GP practice including, but not limited to: liaison at transitions of care, medications information query management, determination of blood samples due and repeat prescriptions management. This list is not finite. The specific focus of the intervention will be the medications optimisation component delivered by targeted patient medication reviews, based on improving safety and addressing national medications management priorities and guidelines. Review of anonymous aggregate data within practices by the pharmacist will also identify specific areas to focus medication optimisation. Medication reviews for consented patients (and practice audit activities) will focus on high-risk prescribing, PIP and deprescribing as per the criteria used in the non-randomised feasibility study. ${ }^{16}$

Patients in the intervention arm will make an appointment with the pharmacist for medication review, preferably in the first 2 months of the intervention. The intervention will run for 4 months in each practice with follow-up data to be collected at completion. Depending on GP availability, further follow-up may be possible after the 4-month intervention period. Figure 2 provides an overview of the process of the GPP intervention.

\section{Control}

Control practices will continue to provide care as usual with no input from the pharmacists. As a form of wait list control, patients in control practices will be offered a medication review with the pharmacist at study completion.

\section{Outcome measures}

The primary outcome of feasibility will include recruitment rate, uptake of medication reviews and study retention. Participant experience will be explored through the process evaluation. We will use continuation criteria (see table 3) to determine if progressing this pilot cRCT to a definitive pragmatic cRCT is warranted based on data from the pilot cRCT and process evaluation.

The primary clinical outcome which will be collected will be the number of PIP incidences per patient, measured at baseline and 4 months. However, a range of 
Practices assessed for eligibility and invited $\mathrm{n}=\mathbf{8}$

- $\geq 500$ patients over the age of 65

- Exclusion criteria: if $<500$ patients over the age of 65 .

Patients recruited from each practice $n=15$ for total of $n=120$ patients

- Polypharmacy ( $\geq 10$ medications)

- $\geq 65$ years of age

- Ambulatory based

- Exclusion criteria; terminally ill, major cognitive impairment, resident in nursing home, currently participating in a related study

\section{Baseline data collection}

- All eight practices

- Informed consent to be obtained prior to data collection

\section{Randomisation (minimisation)}

- Independent researcher blinded to practice details to avoid potential practice level selection bias.

- $\mathrm{n}=4$ intervention arm

- $n=4$ control arm

\section{GPP Medicines Optimisation Programme Intervention Arm}

- Four month intervention period

\section{Control Arm}

- GPs continue to provide care as usual with no input from the research pharmacists

- Intervention to be offered at the end of trial intervention period

\section{Post-intervention Data Collection}

- Matched time points for control and intervention sites

Figure 2 Flow chart for GPP Medicines Optimisation Programme intervention. GP, general practitioner; GPP, General Practice Pharmacist.

medication-related, patient-reported outcome measures (PROMs) and pharmacist impact data will be collected, informed by findings from the non-randomised pilot study, ${ }^{16}$ and core outcome sets for multimorbidity and polypharmacy. ${ }^{19} 20$

Medication-related outcomes from patient's medication record

- Number of PIP per patient, as per criteria used in the non-randomised pilot study and pharmacist clinical judgement. ${ }^{1416}$
- Number of repeat medications.

- Proportion of patients with polypharmacy.

- Medication changes:

- Deprescribing (tapering or stopping) of medications that may cause harm or are no longer providing benefit. ${ }^{21}$

- Medications started.

- Number of medications prescribed generically.

ADRs, defined as an appreciably harmful or unpleasant reaction, resulting from an intervention related to the 
Table 3 Continuation criteria which indicate whether to proceed with a randomised controlled trial (RCT)

\begin{tabular}{|c|c|c|}
\hline Proceed with RCT & $\begin{array}{l}\text { Proceed with RCT following some } \\
\text { changes to the protocol }\end{array}$ & $\begin{array}{l}\text { Do not proceed with RCT unless } \\
\text { problems can be overcome }\end{array}$ \\
\hline Recruitment of 8 general practices & Recruitment of 6-7 general practices & $\begin{array}{l}\text { Unable to recruit at least } 6 \text { general } \\
\text { practices }\end{array}$ \\
\hline $\begin{array}{l}\text { Retention of } \geq 7 \text { general practices } \\
\text { throughout intervention period }\end{array}$ & $\begin{array}{l}\text { Retention of } 6 \text { general practices } \\
\text { throughout intervention period }\end{array}$ & $\begin{array}{l}\text { Retention of } \leq 5 \text { general practices } \\
\text { throughout intervention period }\end{array}$ \\
\hline Recruitment of 1 pharmacist & Recruitment of 1 pharmacist & Unable to recruit 1 pharmacist \\
\hline $\begin{array}{l}\text { Retention of }>12 \text { study patients for } \\
\text { follow-up from each practice }\end{array}$ & $\begin{array}{l}\text { Retention of } 10-<12 \text { study patients for } \\
\text { follow-up from each practice }\end{array}$ & $\begin{array}{l}\text { Retention of }<10 \text { study patients for } \\
\text { follow-up from each practice }\end{array}$ \\
\hline $\begin{array}{l}\text { GPP intervention acceptable to majority } \\
\text { of GPs, pharmacists and patients } \\
\text { involved, as per process evaluation }\end{array}$ & $\begin{array}{l}\text { GPP intervention acceptable to some } \\
\text { GPs, pharmacists and patients involved, } \\
\text { as per process evaluation }\end{array}$ & $\begin{array}{l}\text { GPP intervention acceptable to none } \\
\text { of the GPs, pharmacists and patients } \\
\text { involved, as per process evaluation }\end{array}$ \\
\hline $\begin{array}{l}\text { GPP intervention demonstrates cost- } \\
\text { effectiveness }\end{array}$ & $\begin{array}{l}\text { GPP intervention demonstrates potential } \\
\text { for cost-effectiveness }\end{array}$ & $\begin{array}{l}\text { GPP intervention does not demonstrate } \\
\text { potential for cost-effectiveness }\end{array}$ \\
\hline
\end{tabular}

GPP, General Practice Pharmacist; GPs, general practitioners.

use of a medicinal product, which predicts hazard from future administration and warrants prevention or specific treatment, or alteration of the dosage regimen, or withdrawal of the product. ${ }^{22}$

- Adverse drug withdrawal events, defined as either recurrence of the condition for which the medication was prescribed or a physiological reaction to medication withdrawal. ${ }^{23} 24$

\section{Patient-reported outcome measures}

- Health-related Quality of Life (EQ-5D-5L) ${ }^{25}$

- Revised Patients' Attitudes Towards Deprescribing (rPATD) ${ }^{26}$

- Multimorbidity Treatment Burden Questionnaire. ${ }^{27}$

\section{Role and impact of pharmacists}

- Activities undertaken and total time to complete.

- Condition-specific domains.

- Engagement with other healthcare professionals and practice staff and time spent on each interaction with:

- GPs.

- Community pharmacists.

- Other healthcare professionals/practice staff.

\section{Data collection}

Patient data will be collected at baseline, prior to randomisation, and at intervention completion (4 months after baseline). Medication-related data will be collected from patient's medication records (PMRs). For intervention practices, these records will be reviewed at baseline once the practice has been randomised to the intervention arm, and then at intervention completion. For control practices, records will be reviewed in the PMR at trial completion using previous 'current prescriptions' at baseline and all prescription data for the study period to provide contemporaneous medication data. To ensure the correct baseline prescription has been selected, researchers can review prescriptions issued prior to the baseline data, but that were contemporaneous for the patient at that time.

PROMs will be collected from patient questionnaires and will include the following validated measures: EQ-5D-5L, ${ }^{25}$ burden of treatment ${ }^{28}$ and rPATD. ${ }^{26}$ PROMs data will be collected from control practice patients at matched time points. The variability, consistency, response rates and data completeness for each outcome will be determined.

Data on the role and impact of the pharmacist will be recorded by the practice pharmacists while integrated within the intervention practices using prespecified reporting pharmacist activity log which will record:

1. Activity that the pharmacist undertook.

2. Total time spent on activities, for example, $30 \mathrm{~min}$ spent on audit. This will allow for content analysis and breakdown of time spent on each activity.

3. Engagement and time spent with GP.

4. Engagement and time spent with community pharmacists.

5. Engagement and time spent with other practice staff and healthcare professionals.

6. Condition-specific domains for audit, for example, PIP would be 'safer prescribing'.

7. A 'free text' option which will allow the pharmacist to note interesting observations which they may forget during the qualitative interview at intervention completion.

\section{Sample size}

While no formal sample size is required for a pilot study, based on data we have collected previously, we have 
estimated a sample size which we feel will be large enough to inform about the practicalities of delivering the intervention, recruitment and retention rates, in addition to exploring potential change in PIP outcomes for going forward to a definitive cRCT.

Based on reducing the mean number of PIP per patient from 0.8 by $20 \%$ to 0.64 , using an SD of 0.2 (based on data from the OPTI-SCRIPT Study, ${ }^{29}$ a previous RCT in a similar population) with $90 \%$ power requires a sample size of 68 patients. Based on an intraclass correlation coefficient of 0.025 (OPTI-SCRIPT Study), and 12 patients per practice results in 84 patients from seven practices. We will further inflate this to 120 patients from eight practices to account for loss to follow-up for the PROM secondary outcomes and to account for uncertainty given that these are estimates from different but related populations. In total, we will recruit 120 patients from eight GP practices. This sample size also reflects our experience of patient response rates for participation in these studies and that it is feasible to recruit 15 patients per practice on 10 or more medications. This study will help to establish future sample size calculations that can be used to inform a definitive cRCT.

\section{Blinding}

The trial statistician will be blinded to practice (and thus patient) allocation. Blinding of participant GP practices, patients and pharmacists will not be possible due to the nature of the intervention.

\section{Data analysis}

Descriptive statistics and estimation using CIs will be the main focus of the analysis. Descriptive statistics will be presented for all feasibility outcomes and demographic and clinical characteristics of study participants and practices. For categorical measures, frequencies and percentages will be presented, and for continuous measures, the mean and SD will be reported. For continuous measures which show evidence of some skew, a median and IQR may also be presented or substituted for the mean and SD.

While this study is not powered to explore effectiveness, we will estimate and explore potential differences in all outcome measures, in particular, the difference in the mean number of PIP medications, and the presence/ absence of PIP medications, for the intervention group versus the control, with transformation as appropriate after examination of the distribution, and adjusting for baseline PIP and minimisation factors. Random effects linear or logistic regression models will be used as appropriate, including a random practice effect to account for the correlation between patients in practices. Results will be presented as the difference in means or ORs, $95 \%$ CIs and $p$ values. Other outcomes will be explored and analysed using similar methods.

The analysis of this study will help inform future sample size calculations.

\section{Process evaluation}

To explore participant attitudes towards the intervention and the experience of the intervention delivery, a mixedmethods process evaluation combining both quantitative and qualitative methods will be conducted in line with the MRC Framework for the Process Evaluation of Complex Interventions. ${ }^{30}$ This framework includes an assessment of treatment fidelity of the intervention, which will be incorporated into the study design of the process evaluation study. The Bellg treatment fidelity framework ${ }^{31}$ will be used to monitor and enhance the validity and reliability of interventions involving behavioural change.

Quantitative data will be compiled from completed validated questionnaires, and activity logs which will be summarised with descriptive statistics.

Qualitative data will be collected using semistructured interviews with key stakeholders in the intervention process: GPs, pharmacists, nurses, practice managers and patient participants. A topic guide will be developed to explore the issues surrounding integration, context, fidelity, implementation and experiences of the intervention. Interviews will take approximately $30-60 \mathrm{~min}$ to complete. As it is feasible that pharmacists could increase GP workload, we will explore this issue qualitatively while interviewing GPs. Interviews will take place as per participant preference, either in person or on the phone and they will be recorded to allow for transcription, with participant permission. Data will be transcribed verbatim from the recordings following pseudonymisation of participant identifiers with a unique participant study code. Thematic analysis will be undertaken. ${ }^{32}$ Data will be coded and common features will be grouped to develop themes. Overarching themes will then be developed with quotations used as exemplars. Following analysis, triangulation will be employed to combine the results of both quantitative and qualitative components.

\section{Health economic analysis}

The 'Guidelines for the Economic Evaluation of Health Technologies in Ireland' will be used to inform the economic evaluation component. ${ }^{33}$ The direct costs associated with the intervention will be the focus of the evaluation, and will adopt a payer perspective. The health economic evaluation will focus on calculating direct costs related to the intervention, that is, the costs of recruitment, training and salary of the pharmacist, time/salary of GPs and associated staff, revenue and capital overheads, and travel expenses for pharmacists. In addition, the total costs associated with clinical actions linked to the medication reviews will be calculated including the costs of medications prescribed or deprescribed, cost of laboratory monitoring tests that are recommended, costs saved or incurred. Data to be collected include participants' healthcare utilisation (from patient healthcare records). Unit costs will be applied to convert data on resource use to resource costs and total cost variables will be calculated.

A cost-effectiveness analysis will be conducted on the basis of the primary and secondary outcomes identified in 
the cRCT, that is, reduction in number of PIPs/high-risk prescriptions, deprescribing and pharmacist time. The primary analysis will evaluate the impact of the placement of a pharmacist in a GP practice for a 4-month period. Costs associated with salary, revenue, capital and travel will be informed directly by our 4-month trial data. Inflation to the 12-month time horizon will allow us to investigate potential longer term sequela avoided due to the PIP intervention made by the pharmacists. It is assumed that the changes made to a patients' long-term medication (as a result of a pharmacist intervention) will persist beyond the 4-month intervention period. In line with this, costs incurred or saved secondary to the medication reviews will be informed by our 4-month trial data and inflated to a 12-month time horizon. A scenario analysis will evaluate the impact of the placement of a pharmacist in a GP practice for a 12-month period. Costs associated with salary, revenue, capital and travel will be informed directly by our trial data and inflated to a 12-month time horizon. The expected continued rate of pharmacists' interventions beyond the 4-month time period (available from our data) will be extrapolated to 12 months using data published elsewhere and via expert elicitation. Costs incurred or saved secondary to medication reviews will be informed by these extrapolated data. The incremental cost per PIP intervention will be estimated for both the primary and scenario analyses. The impact on changes made to all uncertain model input parameters will be investigated in both one-way sensitivity analysis and probabilistic sensitivity analysis.

The cost-effectiveness analysis will allow us to consider the potential data requirements for a lifetime horizon in future cost-effectiveness analyses, if a definitive cRCT is carried out. This would allow us to investigate the longterm sequela avoided due to PIP intervention, giving consideration to patient or therapeutic subgroups where there is established evidence between PIP and ADR-related hospital admissions (eg, non-steroidal antiinflammatory drugs in people with heart failure and admissions for exacerbation). Within the process evaluation, we will employ expert elicitation regarding potential wider effects of the intervention on prescribing practice to inform model assumptions.

\section{Continuation criteria}

We will use continuation criteria (see table 3) to determine if progressing this pilot cRCT to a definitive pragmatic cRCT is warranted. The criteria for continuation (also referred to as progression criteria) will be based around feasibility and the potential for cost-effectiveness. Quantitative and qualitative process evaluation data will be analysed to consider the following continuation criteria:

- Recruitment levels of GP practices, pharmacists and patients.

- Acceptability of intervention for GPs, pharmacists and patients.

- Feasibility of GPP intervention.
Potential to be cost-effective.

Ethics, data management and dissemination

Ethical approval has been granted by the Irish College of General Practitioners Research Ethics Committee. In the event that a prescribing pattern of concern is identified by the research team, as a safeguard this will be referred to an external, independent clinician who will have no involvement in the study to advise the team on appropriate actions.

Patient healthcare records will be accessed to obtain prescription data and healthcare utilisation data. PROM data will be collected from paper-based completed patient questionnaires. For the process evaluation, data will be collected by the verbatim transcription of audio recordings of semistructured interviews. Transcription of audio recordings will be done by a third party with whom we will have a confidentiality agreement, and patient consent will be obtained for this.

Data will be pseudonymised at practice level. Participants will be known to the research team by study ID number only. One member of the research team (AC) will have access to patient contact details for follow-up data collection purposes. Required contact details will be linked to the study number and stored in a restricted access folder on a secure institutional server. Hard copies of data such as questionnaires will be stored in locked cabinets in the university research offices which are accessible only by swipe card access.

The results will be reported in peer-reviewed journals and be presented at national and international conferences.

\section{Patient and public involvement}

This study has patient and public (PPI) involvement through a multimorbidity patient advisory group. The patient advisory group are patients with multimorbidity who meet quarterly to discuss issues arising with research projects on multimorbidity funded through the HRB Collaborative Doctoral Award (AC is a PhD student on this programme). This PPI group was asked to contribute to the development and refinement of this intervention, as outlined in table 1 .

\section{DISCUSSION}

Polypharmacy is a contributing factor to the treatment burden associated with multimorbidity. PIP is associated with complex polypharmacy and studies have shown that this leads to medical complications and associated costs. ${ }^{7}$ A wide variety of interventions have been developed to address the issue of polypharmacy and PIP in primary care settings but the evidence base for the integration of pharmacists into GP practices is limited as few highquality RCTs, and modest effect sizes have been reported. A number of large trials have been conducted in this area in other jurisdictions, for example, Canada, ${ }^{34}$ the UK, ${ }^{12}$ Australia, ${ }^{35}$ New Zealand ${ }^{36}$ and Sweden. ${ }^{37}$ Pharmacists 
have direct prescribing roles in the UK, New Zealand and Canada, but similar to the Irish context, pharmacists in Australia and Sweden do not. Quality of life measures by these trials produced varying results. ${ }^{36}$ Irrespective of prescribing roles, all trials were broadly similar, adopting a model of patient medication review and quality enhancement in practice. Our own trial also has similar interventions of patient medication review and practice-level education and quality enhancement. This suggests that our results will be generalisable to other health jurisdictions. High-quality trials such as PINCER demonstrated a positive effect using a pharmacist-led information technology intervention to address medication errors. ${ }^{12}$ Our trial seeks to build on this experience in a health system with less integration of pharmacists in general practice settings. The outcomes of a non-randomised, uncontrolled feasibility study ${ }^{16}$ have been reported and based on predefined continuation criteria the trial is being progressed to further evaluation with the design of this pilot cRCT. We have reported here the protocol for the pilot cRCT. If the pilot cRCT is feasible to conduct, acceptable to stakeholders, and demonstrates a positive impact and cost-effectiveness, we will aim to proceed to a full, pragmatic cRCT. With the increased medicalisation of managing conditions, the need for interventions to address polypharmacy and PIP is warranted.

Recruitment of GPs may be a potential challenge, however feedback from the feasibility study was positive. We will seek the assistance of the HRB CTNI to support this process. Eligibility criteria for GP practices dictate that there must be $\geq 500$ patients aged 65 years or older for the practice to be considered. To allow for feasible delivery of the intervention, allocation of practices will be done in blocks to allow for staggered data collection. Logistical barriers will be addressed with practices to ensure that the pharmacist has access to an office, or space in an office, with access to the practice clinical software to complete their intervention-related activity. It is envisaged that the pharmacist will allocate a single block of time, however, these working practices are flexible to allow for successful integration of the pharmacist into the GP practice. As the study is designed as a pilot cRCT, a power calculation is not required. However, we have calculated that the number of patients recruited will give sufficient power to detect a significant change in PIP. Given the relatively small number of study participants the results will not be generalisable, and the primary purpose of the pilot cRCT is to test feasibility and identify appropriate processes, outcomes and sample size calculation parameters for a definitive cRCT.

In summary, the GPP Medicines Optimisation Programme aims to assess the feasibility and potential impact of general practice-based pharmacists in an Irish setting in relation to addressing PIP and GP workload.

Twitter Aisling Croke @AislingCroke1 and Susan M Smith @susanmsmithm

Contributors $A C$ and $B C$ drafted the manuscript. AC, FM, FB, LM, KC, SMS and $\mathrm{BC}$ were involved in the study design and in obtaining ethical approvals. SMS was responsible for study conception. All authors read, provided feedback and approved the final manuscript.

Funding This research was supported by the Health Research Board Collaborative Doctoral Award (CDA-2018-003).

Competing interests None declared.

Patient consent for publication Not required.

Provenance and peer review Not commissioned; externally peer reviewed.

Supplemental material This content has been supplied by the author(s). It has not been vetted by BMJ Publishing Group Limited (BMJ) and may not have been peer-reviewed. Any opinions or recommendations discussed are solely those of the author(s) and are not endorsed by BMJ. BMJ disclaims all liability and responsibility arising from any reliance placed on the content. Where the content includes any translated material, BMJ does not warrant the accuracy and reliability of the translations (including but not limited to local regulations, clinical guidelines, terminology, drug names and drug dosages), and is not responsible for any error and/or omissions arising from translation and adaptation or otherwise.

Open access This is an open access article distributed in accordance with the Creative Commons Attribution Non Commercial (CC BY-NC 4.0) license, which permits others to distribute, remix, adapt, build upon this work non-commercially, and license their derivative works on different terms, provided the original work is properly cited, appropriate credit is given, any changes made indicated, and the use is non-commercial. See: http://creativecommons.org/licenses/by-nc/4.0/.

ORCID iDs

Fiona Boland http://orcid.org/0000-0003-3228-0046

Susan M Smith http://orcid.org/0000-0001-6027-2727

Barbara Clyne http://orcid.org/0000-0002-1186-9495

\section{REFERENCES}

1 Nguyen H, Manolova G, Daskalopoulou C, et al. Prevalence of multimorbidity in community settings: a systematic review and meta-analysis of observational studies. J Comorb 2019;9:2235042X19870934-2235042X

2 Moriarty F, Hardy C, Bennett K, et al. Trends and interaction of polypharmacy and potentially inappropriate prescribing in primary care over 15 years in Ireland: a repeated cross-sectional study. BMJ Open 2015;5:e008656.

3 Hughes LD, McMurdo MET, Guthrie B. Guidelines for people not for diseases: the challenges of applying UK clinical guidelines to people with multimorbidity. Age Ageing 2013;42:62-9.

4 Hedna K, Hakkarainen KM, Gyllensten $\mathrm{H}$, et al. Potentially inappropriate prescribing and adverse drug reactions in the elderly: a population-based study. Eur J Clin Pharmacol 2015;71:1525-33.

5 Cooper JA, Cadogan CA, Patterson SM, et al. Interventions to improve the appropriate use of polypharmacy in older people: a Cochrane systematic review. BMJ Open 2015;5:e009235.

6 Kaufmann CP, Tremp R, Hersberger KE, et al. Inappropriate prescribing: a systematic overview of published assessment tools. Eur J Clin Pharmacol 2014;70:1-11.

7 Cahir C, Fahey T, Teeling M, et al. Potentially inappropriate prescribing and cost outcomes for older people: a national population study. Br J Clin Pharmacol 2010;69:543-52.

8 Rankin A, Cadogan CA, Patterson SM, et al. Interventions to improve the appropriate use of polypharmacy for older people. Cochrane Database Syst Rev 2018;37:Cd008165.

9 Clyne B, Fitzgerald C, Quinlan A, et al. Interventions to address potentially inappropriate prescribing in community-dwelling older adults: a systematic review of randomized controlled trials. J Am Geriatr Soc 2016;64:1210-22.

10 Tan ECK, Stewart K, Elliott RA, et al. Pharmacist services provided in general practice clinics: a systematic review and meta-analysis. Res Social Adm Pharm 2014;10:608-22.

11 Anderson C, Zhan K, Boyd M, et al. The role of pharmacists in general practice: a realist review. Res Social Adm Pharm 2019;15:338-45.

12 Avery AJ, Rodgers S, Cantrill JA, et al. A pharmacist-led information technology intervention for medication errors (pincer): a multicentre, cluster randomised, controlled trial and cost-effectiveness analysis. Lancet 2012;379:1310-9.

13 Hayhoe B, Cespedes JA, Foley K, et al. Impact of integrating pharmacists into primary care teams on health systems indicators: a systematic review. Br J Gen Pract 2019;69:e665-74. 
14 Cardwell K, Clyne B, Moriarty F, et al. Supporting prescribing in Irish primary care: protocol for a non-randomised pilot study of a general practice pharmacist (Gpp) intervention to optimise prescribing in primary care. Pilot Feasibility Stud 2018;4:122.

15 James O, Cardwell K, Moriarty F, et al. Pharmacists in general practice: a qualitative process evaluation of the general practice pharmacist (Gpp) study. Fam Pract 2020;37:cmaa044

16 Cardwell K, Smith SM, Clyne B, et al. Evaluation of the general practice pharmacist (Gpp) intervention to optimise prescribing in Irish primary care: a non-randomised pilot study. BMJ Open 2020;10:e035087.

17 Eldridge SM, Chan CL, Campbell MJ, et al. Consort 2010 statement: extension to randomised pilot and feasibility trials. BMJ 2016;355:i5239.

18 Craig P, Dieppe P, Macintyre S, et al. Developing and evaluating complex interventions: the new medical Research Council guidance. BMJ 2008;337:a1655

19 Rankin A, Cadogan CA, in Ryan C, et al. Core outcome set for trials aimed at improving the appropriateness of polypharmacy in older people in primary care. J Am Geriatr Soc 2018;66:1206-12.

20 Smith SM, Wallace E, Salisbury C, et al. A core outcome set for multimorbidity research (COSmm). Ann Fam Med 2018;16:132-8.

21 Scott IA, Hilmer SN, Reeve E, et al. Reducing inappropriate polypharmacy: the process of deprescribing. JAMA Intern Med 2015;175:827-34.

22 Edwards IR, Aronson JK. Adverse drug reactions: definitions, diagnosis, and management. Lancet 2000;356:1255-9.

23 Gerety MB, Cornell JE, Plichta DT, et al. Adverse events related to drugs and drug withdrawal in nursing home residents. J Am Geriatr Soc 1993;41:1326-32.

24 Graves T, Hanlon JT, Schmader KE, et al. Adverse events after discontinuing medications in elderly outpatients. Arch Intern Med 1997;157:2205-10.

25 EuroQol G, EuroQol Group. EuroQol--a new facility for the measurement of health-related quality of life. Health Policy 1990;16:199-208.

26 Reeve E, Low L-F, Shakib S, et al. Development and validation of the revised patients' attitudes towards deprescribing (rPATD) questionnaire: versions for older adults and caregivers. Drugs Aging 2016;33:913-28.
27 Duncan P, Murphy M, Man M-S, et al. Development and validation of the multimorbidity treatment burden questionnaire (MTBQ). BMJ Open 2018;8:e019413.

28 May CR, Eton DT, Boehmer K, et al. Rethinking the patient: using burden of treatment theory to understand the changing dynamics of illness. BMC Health Serv Res 2014;14:281.

29 Clyne B, Smith SM, Hughes CM, et al. Effectiveness of a multifaceted intervention for potentially inappropriate prescribing in older patients in primary care: a cluster-randomized controlled trial (OPTI-SCRIPT study). Ann Fam Med 2015;13:545-53.

30 Moore GF, Audrey S, Barker M, et al. Process evaluation of complex interventions: medical Research Council guidance. BM 2015;350:h1258

31 Bellg AJ, Borrelli B, Resnick B, et al. Enhancing treatment fidelity in health behavior change studies: best practices and recommendations from the NIH behavior change Consortium. Health Psychol 2004;23:443-51.

32 Braun V, Clarke V. Using thematic analysis in psychology. Qual Res Psychol 2006;3:77-101.

33 Health Information and Quality Authority. Guidelines for the economic evaluation of health technologies in Ireland. Dublin: HIQA, 2019.

34 Al Hamarneh YN, Hemmelgarn BR, Hassan I, et al. The Effectiveness of Pharmacist Interventions on Cardiovascular Risk in Adult Patients with Type 2 Diabetes: The Multicentre Randomized Controlled R EACH Trial. Can J Diabetes 2017;41:580-6.

35 WentWest. Western Sydney general practice pharmacist program: integrating pharmacists into the patient care team. Western Sydney: Public Health Network,Australian Government, 2018.

36 Bryant LJM, Coster G, Gamble GD, et al. The general PractitionerPharmacist collaboration (GPPC) study: a randomised controlled trial of clinical medication reviews in community pharmacy. Int $J$ Pharm Pract 2011;19:94-105.

37 Lenander C, Elfsson B, Danielsson B, et al. Effects of a pharmacistled structured medication review in primary care on drug-related problems and hospital admission rates: a randomized controlled trial. Scand J Prim Health Care 2014;32:180-6.

38 Pharmacy act, 2007. Available: http://www.irishstatutebook.ie/eli/ 2007/act/20/enacted/en/html [Accessed Feb 20, 2021]. 